\title{
PERFORMANCE INDICATORS FOR QUALITY IN SURGICAL AND LABORATORY SERVICES AT MUHIMBILI NATIONAL HOSPITAL (MNH) IN TANZANIA
}

\author{
Naboth A.Mbembati ${ }^{1}$, Mugwira Mwangu ${ }^{2}$, Eustace P.Y. Muhondwa ${ }^{3}$, Melkizedek M. Leshabari ${ }^{3}$
}

\section{Abstract:}

Objectives: Muhimbili National Hospital (MNH), a teaching and national referral hospital, is undergoing major reforms to improve the quality of health care. We performed a retrospective descriptive study using a set of performance indicators for the surgical and laboratory services of MNH in years 2001 and 2002, to help monitor and evaluate the impact of reforms on the quality of health care during and after the reform process.

Methodology: Hospital records were reviewed and information recorded for planned and postponed operations, laboratory equipment, reagents, laboratory tests and quality assurance programmes.

Results: In the year 2001 a total of 4332 non-emergency operations were planned, 3313 operations were performed and 1019 (23.5\%) operations were postponed. In the year 2002, 4301 non-emergency operations were planned, 3046 were performed and 1255 (29\%) were postponed. The most common reasons for operation postponement were "time-barred", interference by emergency operations, no show of patients and inoperable anaesthetic machines. Equipment problems and supply and staff shortages together accounted for one quarter of postponements. In the laboratory, a lack of equipment prevented some tests, but quality assurance was performed for most tests.

Conclusion: Current surgical services at MNH are inadequate; operating theatres require modern, functioning equipment and adequate supplies of consumables to provide satisfactory care

Keywords: Referral hospital, performance indicators, surgical services, laboratory services

\section{Introduction}

Muhimbili National Hospital (MNH) is the national reference and teaching hospital in Tanzania. Due to its location, MNH also acts as a referral hospital for the city of Dar es Salaam and receives patients from all health units in the city. The hospital is currently undergoing a major reform process that includes infrastructural changes focused on improving the quality of health care and the introduction of quality assurance monitoring. These changes involve reducing bed capacity from 1500 to about 800 beds.

This study commenced with an evaluation of the functioning of $\mathrm{MNH}$. The WHO hospital advisory committee provides guidelines for the establishment of performance indicators for referral hospitals (1). However, which performance indicators one uses depends on the level of care of the institution, the level of sophistication of the unit and the volume or workload. For example large operating suites with large surgical outputs may wish to use Operating Room utilization rates and turn over times between cases (2). Others include cancellation rates, start time accuracy, patient, staff and employee satisfaction and cost per case (3). Similarly for laboratories the following indicators have been used: order accuracy rates, laboratory testing error, laboratory reporting error, interpretation misjudgments in microbiology (4).

Evaluation of the performance of MNH coincides with commencement of rehabilitation to provide baseline information for the improvement of the quality of health care. Performance indicators are required for the evaluation and continued monitoring of surgical and laboratory services, the two essential services offered by $\mathrm{MNH}$ (5).

Correspondence to: N. Mbembati, P. O. Box 65001, Muhimbili University of Health and Allied Sciences, Dar es Salaam - Tanzania.: E-mail: nmbembati@muhas.ac.tz

${ }^{1}$ Dept of Surgery, Muhimbili University of Health \& Allied Sciences (MUHAS); ${ }^{2}$ Dept of Development Studies; ${ }^{3}$ Dept of Behavioural Sciences, MUHAS
This is important as patients will be attracted to a hospital providing good quality surgical services and laboratory services which provide the framework for almost all other clinical departments.

$\mathrm{MNH}$ has adequate staff in all major medical and surgical disciplines. Furthermore, medical school teachers who also offer clinical services, add to the hospital workforce. We performed a retrospective study of performance in the surgical department and laboratory services of $\mathrm{MNH}$ to provide a baseline and framework for ongoing monitoring and evaluation during and after the implementation of the reform process.

\section{Methods}

Data were collected by research assistants who completed a series of checklists using hospital records. All research assistants underwent training on the process of data collection and how to fill in the checklists. To evaluate surgical services, information on planned and postponed operations was obtained, including reasons for postponement of surgery, from a well-kept theatre nurses register. The records of 1566 patients whose operations were postponed were retrieved to survey the underlying reasons for postponement during 2001 and 2002.

To evaluate laboratory services, the amount of downtime of technical equipment/year, number of reagents out of stock/year, proportion of requested tests available, and proportion of all laboratory tests having a quality assurance program were recorded.

The data were analyzed after having been entered in Excel spreadsheets.

\section{Results}

In the year 2001, a total of 4332 non-emergency operations were planned, 3313 operations were performed and $1019(23.5 \%)$ operations were postponed. In the year 
2002, 4301 non-emergency operations were planned, 3046 were performed and $1255(29 \%)$ were postponed (See Table $1 \mathrm{a}$ and $1 \mathrm{~b})$.

The results from the survey of records of patients whose operations were postponed are summarized in Table 2. The most common causes of postponement were "time barred", interference by emergency and no show of patients (patient not in the ward). Equipment problems, particularly anaesthetic machines being out of order, materials and consumables supply, and staff shortages together accounted for one quarter of postponements.

Table 1a: Planned, operated and postponed patients in 2001

\begin{tabular}{lllll}
\hline Month & Planned & Operated & Postponed & \% Postponed \\
\hline January & 359 & 287 & 72 & 20.0 \\
February & 325 & 238 & 87 & 26.8 \\
March & 334 & 246 & 88 & 26.3 \\
April & 352 & 250 & 102 & 28.9 \\
May & 351 & 275 & 76 & 21.6 \\
June & 380 & 290 & 90 & 23.7 \\
July & 419 & 317 & 102 & 24.3 \\
August & 340 & 270 & 70 & 20.6 \\
September & 366 & 296 & 70 & 19.1 \\
October & 376 & 289 & 87 & 23.1 \\
November & 350 & 253 & 97 & 27.7 \\
December & 380 & 302 & 78 & 20.5 \\
\hline Total & $\mathbf{4 3 3 2}$ & $\mathbf{3 3 1 3}$ & $\mathbf{1 0 1 9}$ & $\mathbf{2 3 . 5}$ \\
\hline
\end{tabular}

Table 1b: Planned, operated and postponed patients in 2002

\begin{tabular}{lllll}
\hline Month & Planned & Operated & Postponed & \% Postponed \\
\hline January & 372 & 289 & 83 & 22.3 \\
February & 361 & 258 & 103 & 28.5 \\
March & 377 & 236 & 141 & 37.4 \\
April & 329 & 218 & 111 & 33.7 \\
May & 370 & 255 & 115 & 31.1 \\
June & 441 & 268 & 173 & 39.2 \\
July & 337 & 262 & 75 & 22.2 \\
August & 331 & 220 & 111 & 33.5 \\
September & 420 & 325 & 95 & 22.6 \\
October & 326 & 250 & 76 & 23.3 \\
November & 329 & 240 & 89 & 27.1 \\
December & 308 & 225 & 83 & 26.9 \\
\hline Total & $\mathbf{4 3 0 1}$ & $\mathbf{3 0 4 6}$ & $\mathbf{1 2 5 5}$ & $\mathbf{2 9 . 2}$ \\
\hline
\end{tabular}

During 2001 and 2002 laboratory services in the Department of Haematology provided haemoglobin estimations, full blood picture (FBP), erythrocyte segmentation rate (ESR), prothrombin time (PT), partial thromboblastin time (PTT) analyses, and bone marrow biopsies and did not report breakdown of equipment. A lack of equipment in this department did however prevent analyses of bleeding and clotting times. The Department of Biochemistry tested blood sugar, serum creatinine, blood urea, alanine aminotransferase (ALT), aspartate aminotransferase (AST) and serum alkaline phosphatase. Serology testing for HIV was also performed. While there was no report of equipment breakdown a lack of equipment prevented the performance of hepatitis BsAg and blood Widal tests. Similarly, the Department of Microbiology did not report breakdown of equipment and performed Gram staining; pyogenic and mycobacterial tuberculosis cultures. The Department of Parasitology performed analyses of urine, stools and blood slide for parasites (malaria and microfilaria) without reporting breakdown of equipment and the Department of Pathology performed histological and cytological examinations without any technical problems or breakdown of equipment.

The monitoring of reagent availability was similar in all laboratory departments and all departments used the same source of reagents. The head laboratory technician maintained supply of materials and consumables by comparing the number of tests done against the supply of reagents available and ordering new stock one week in advance. Apart from tests that were not performed due to lack of equipment, including tests for bleeding and clotting time, serum electrolytes, prostate specific antigen (PSA), and thyroid hormone levels, there was no canceling of tests due to a lack of supply of reagents or stock during the two year duration of the study.

Quality assurance was performed daily for the following tests: HB, Blood Sugar, Creatinine, Urea, Serum Bilirubin, ALT, AST Alkaline Phosphatase, HIV, ZN staining and Mycobacterium TB culture. Weekly quality assurance was performed for histology and cytology. Quality assurance was not performed for urine or stool analyses, blood slides for malaria parasites or microfilaria or for tests not conducted in the laboratory.

With the exception of histology all tests performed at the MNH or other Dar es Salaam hospitals and the records do not specify the location of the testing. Most of the requests for histology testing were from $\mathrm{MNH}$ with about $20 \%$ or the requests originating from other Dar es Salaam city hospitals (Table 3 )

Table 2: Categorization of reasons for postponement of planned operations.

\begin{tabular}{|c|c|c|c|}
\hline & Reason & Number & $\%$ \\
\hline 1 & $\begin{array}{l}\text { Organizational problems } \\
\text { Patient "time barred", interference by } \\
\text { emergencies }\end{array}$ & 794 & 52.6 \\
\hline 2 & $\begin{array}{l}\text { Patient related problems } \\
\text { High } B P \text {, low } H B \text {, no consent, no show }\end{array}$ & 285 & 18.9 \\
\hline 3 & $\begin{array}{l}\text { Technical problem with equipment } \\
\text { Out of order autoclaves, anaesthetic } \\
\text { machines and laundry machines }\end{array}$ & 193 & 12.8 \\
\hline 4 & $\begin{array}{l}\text { Shortage of materials and consumables } \\
\text { e.g. linen, blood, water for irrigation }\end{array}$ & 153 & 10.1 \\
\hline 5 & $\begin{array}{l}\text { Shortage of staff } \\
\text { Doctor not available due to other duties }\end{array}$ & 40 & 2.7 \\
\hline 6 & $\begin{array}{l}\text { Others (one or two patients each) } \\
\text { No Cidex (Glutaraldehyde), patient too } \\
\text { obese, no appropriate cuff to take BP }\end{array}$ & 44 & 2.9 \\
\hline & Total & 1509 & 100.0 \\
\hline
\end{tabular}

Organizational problems, poor patient preparation and break down of equipment were the most common causes of postponement of surgery. 
Table 3: Distribution of source of requests for histology for 2001 and 2002

\begin{tabular}{rrlrrr}
\hline & \multicolumn{1}{l}{ MNH } & \multicolumn{1}{l}{ DSM } & \multicolumn{1}{l}{ Upcountry } & \multicolumn{1}{l}{ Total } \\
\hline & 2001 & $2148(79.5 \%)$ & $549(20.3 \%)$ & $3(0.1 \%)$ & $2700(99.9 \%)$ \\
200 & 2002 & $2813(69.7 \%)$ & $766(19.0 \%)$ & $454(11.2 \%)$ & $4033(99.9 \%)$ \\
\hline & Total & $4961(73.7 \%)$ & $1315(19.5 \%)$ & $457(6.8 \%)$ & $6733(100.0 \%)$ \\
\hline
\end{tabular}

Most of the histology requests were from Muhimbili National Hospital. About $20 \%$ of the requests come from Dar es Salaam city hospitals.

\section{Discussion}

The evaluation of hospital performance provides an essential framework for evidence-based medical care. A set of parameters for the evaluation and monitoring of the provision of quality medical care is necessary. The WHO hospital advisory committee produced guidelines for the establishment of performance indicators to be used in evaluating health system performance and to determine how well an institution is performing in terms of quality clinical and clinical support services (1). The present study established baseline performance indicators for $\mathrm{MNH}$ in clinical and clinical support areas for evaluation and continued monitoring of care quality. Other African countries are now following to set similar hospital performance indicators (6-8).

The present study identified theatre services at $\mathrm{MNH}$ as unsatisfactory. A postponement rate of planned nonemergency surgeries of $23.5 \%$ in 2001 and 29.2 in 2002 $(25.6 \%$ overall) is considered too high (Table 1). Investigations into the reasons underlying operation postponement reveal that most causes were avoidable and could have been effectively overcome at different levels. Issues readily addressed by management include the maintenance or replacement of dysfunctional air conditioners, anaesthetic machines, and diathermy machines. Other areas could be improved with efficient planning and communication and the aid of adequate and well-motivated staff. Interference of planned nonemergency surgery by emergencies could be overcome by allocating an operating theatre (suite) and a theatre team specifically for emergencies. It is unclear why a patient should appear in an operating list and yet be recorded as "no show". This could be attributed to poor planning or bad communication and overcome with more efficient management, planning and communication. Similarly, "time-barred" could be a result of poor planning. Staff management and efficient time management may improve this cause of postponed surgery. Unexpectedly long cases may contribute to but are unlikely to be a leading cause of postponement. It is possible "time-barred" reflects a shortage of equipment and/or resources. Essential equipment may need to be shared among patients thereby increasing waiting times and increasing the occurrence of "time-barred" as a cause of operational postponement. Similarly, the placement of consumables in one location rather than in small stockpiles in each theatre would increase surgery waiting time.
Inadequate patient evaluation also played a role in increasing the rate of surgery postponement. Patients planned for surgery and subsequently identified as anaemic, hypertensive or febrile due to inadequate investigation need to have planned surgery postponed until their condition improves. The reasons underlying inadequate patient investigation may include carelessness, irresponsibility, low morale, lack of motivation or lack of equipment and/or facilities. It is essential that these issues be addressed and further studies are required to provide answers. Postponement of surgery impacts on different parties and results in "backlogging" of patients on the waiting list, disruption of the patient's plans and those of close family members, increased psychological trauma of the patient. Equally disturbing are the uncomfortable and unnecessary surgical preparations undergone by patients, including colonic washouts or nasogastric tube insertation with lavage prior to patients being "time-barred".

The "no consent", "no show" or "patient did not fast" reasons for postponement of planned surgery, following admission for surgery, are strongly indicative of poor communication between patients and staff.

Surgical services are an important component of a hospital and patients will usually travel long distances in search of quality health care. The issue of poor surgical services should be squarely addressed because theatre service is a very important component of a hospital. Patients will usually travel long distances in search of quality services and will avoid a hospital not offering quality theatre services (2).

This report identified many essential laboratory services that cannot be performed at $\mathrm{MNH}$ and this is inadequate for a national teaching and reference hospital. The Department of Biochemistry was most affected and this is critical as physicians require efficient biochemical analyses for appropriate management of acute medical and surgical illnesses. Problems were not encountered with reagent availability when equipment was available. Quality assurance was not performed for all tests, including urine or stool analyses or blood slide tests for malaria and microfilaria, and some tests were performed daily and others weekly. Implementation of quality assurance consistency and benchmarking is required to improve efficiency and enhance the quality of health care.

In 2001 and 2002 the majority of histology specimens, 79.5 and $67.9 \%$, respectively, originated from $\mathrm{MNH}$. By comparison, only 0.1 and $11.2 \%$ respectively, (73.7\%) originated from upcountry. The disparity in the level of histology specimens originating from upcountry in 2001 and 2002 may be due to errors in record keeping or may be due to the presence of pathologists in Mbeya and Kilimanjaro Christian Medical Centre (KCMC) hospitals (two other zonal referral hospitals).

Limitations of this study include, (1) the unavailability of some record books due to ongoing renovation of the laboratory facilities, (2) the lack of performance of serum electrolytes, thyroid function tests, and bleed and clotting times during the study period due to lack of equipment, (3) the inability to obtain mortality data for major emergency surgery and non emergency surgery which are important performance indicators from the record keeping system 
due to technical difficulties.

Theatre services are inadequate at $\mathrm{MNH}$. In-depth analyses of the reasons for inadequacy tend to point to under funding or lack of resources as the main problem. Overcoming theatre service problems involves equipping the operating theatre with modern equipment, replacing dilapidated equipment and making sure that the necessary basic consumables are constantly available in adequate amounts. Laboratory services at $\mathrm{MNH}$ are similarly inadequate, and basic investigations cannot be performed. Available services pose no problems with quality assurance or availability of reagents. The inability to perform basic laboratory tests has a drastically detrimental effect on the quality of patient medical care. The laboratory should be rehabilitated and equipped with all standard equipment to enable it deliver quality laboratory services commensurate with a level 2 and 3 hospital.

\section{Acknowledgements}

We wish to thank the AXIOS Foundation for financial support for this study. We also thank the Management of Muhimbili National Hospital for their support.

\section{References:}

1. WHO. 1994. A review of determinants of hospital performance, review of hospital advisory group meeting. GenevaWHO/SHS/DHS/94.6.

2. OR benchmark activities for the day of surgery. OR manager, August 2000; 16(8): 1-3.

3. Healthcare Financial Management Association, Comprehensive Performance management in the Operating Room, a synopsis. 2002.

4. Nevalainedn, D; Berte Lucia; Kraft C et al. Evaluating laboraroty Performance on Quality Indicators with the Six Sigma Scale. Arch pathol lab Med 2000; 124:516-519.

5. External Consultant Report: Harmonization reform and rehabilitation at Muhimbili National Hospital, January 2000.

6. Molyneux, E and Weber, MW,. Applying the right standards to import hospital performance in Africa. The Lancet 2004; 364: 1560-1561.

7. Kane-Berman, Taylor, SP. . Containing Coasts in Public sector Hospitals - a strategy for the future; lessons from a large teaching hospital. SAMJ 1990; 78 154-157.

8. English, M, Esamai, F, Wassuna A. et al.. Assessment of inpatient Paediatric care in first referral level hospitals in 13 districts. The Lancet 2004; 363: 19481953.

Received 31 July 2007; Revised 14 February 2008; Accepted 13 March 2008 